\title{
Association Between Force-Time Curve Characteristics AND Vertical Jump Performance in Trained Athletes
}

\author{
Mário C. Marques, ${ }^{1,2}$ Mikel Izquierdo, ${ }^{3}$ Daniel A. Marinho, ${ }^{1,2}$ Tiago M. Barbosa, $, 2,4$ \\ Ricardo Ferraz, ${ }^{1,2}$ and Juan José González-Badillo ${ }^{5}$ \\ ${ }^{1}$ Department of Sport Sciences, University of Beira Interior, Covilha, Portugal; ${ }^{2}$ Research Centre in Sport, Health and Human \\ Development, Vila Real, Portugal; ${ }^{3}$ Department of Health Sciences, Public University of Navarre, Pamplona, Navarre, Spain; \\ ${ }^{4}$ National Institute of Education, Nanyang Technological University, Singapore, Singapore; and ${ }^{5}$ Faculty of Sport, Pablo de \\ Olavide University, Seville, Spain
}

\begin{abstract}
Marques, MC, Izquierdo, M, Marinho, DA, Barbosa, TM, Ferraz, R, and González-Badillo, JJ. Association between force-time curve characteristics and vertical jump performance in trained athletes. J Strength Cond Res 29(7): 2045-2049, 2015-Countermovement jump (CMJ) has been extensively used in training; yet, limited and contradictory kinematic data are available for trained subjects. To our best knowledge, no other studies have evaluated the associations between force-time curve characteristics and CMJ in a large sample of trained athletes using a linear transducer. Thus, the aim of this study was to determine the association between force-time measures and CMJ performance collected with a linear transducer. Thirty-five trained athletes were asked to perform 3 maximal weighted CMJ using a linear transducer attached to a barbell $(17 \mathrm{~kg})$. The data indicated that the maximal rate of force development $\left(\mathrm{RFD}_{\max }\right)$ was strongly related to $\mathrm{CMJ}$ displacement $(r=0.809 / 0.807, p<0.001)$ and also to the percentage of peak force $(r=-0.823 /-0.809, p<$ 0.001 ) at $\mathrm{RFD}_{\max }$. Velocity and displacement at $\mathrm{RFD}_{\max }$ were not correlated to $\mathrm{CMJ}$ height. It was therefore concluded that the percentage of PF applied at RFD ${ }_{\max }$ and $\mathrm{RFD}_{\text {max }}$ were the best predictive variables for CMJ performance in this study.
\end{abstract}

KEY WoRDS maximal rate of force development, peak force, countermovement jump

Address correspondence to Mikel Izquierdo, mikel.izquierdo@gmail.com. $29(7) / 2045-2049$

Journal of Strength and Conditioning Research (C) 2015 National Strength and Conditioning Association

\section{INTRODUCTION}

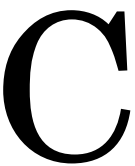
ountermovement jump (CMJ) performance has been studied by the scientific community for many years $(1,5,6,21)$. This motor skill is a wellrounded way to monitor explosive strength and assess the response to different training programs $(2,3,18,26)$.

So far, several experiments have identified multiple factors that play a role on the CMJ performance $(1-4,26)$. Dowling and Vamos (11) examined 15 parameters in roughly 100 trained and untrained subjects of both genders and reported that the positive peak power was a strong predictor of vertical jump performance. Aragon-Vargas and Gross (2) verified that takeoff velocity explained approximately $60 \%$ of the variance in jump height. Nevertheless, findings are equivocal as to which mechanical variables best predict $\mathrm{CMJ}$ performance $(2,3)$. Yet, there are mixed findings about jump performance in distinct populations $(10,22)$, such as fairly small sample power and participants' characteristics. In addition, few studies have examined $\mathrm{CMJ}$ performance determinants in large samples of trained athletes $(12,20,24)$.

Several authors $(9,12,27)$ have claimed that measurements of maximum force (i.e., maximum isometric force 1 repetition maximum) are not indicative of the contractile properties of a muscle. Indeed, force capabilities of muscles should be examined under conditions depending on time, motion speed, and acceleration (1,6). Accordingly, explosive strength is defined as the ability to produce large force or torque values in a limited period of time with high rates of force development (RFDs) and has been one of the most important variables to explain performance in activities where great acceleration is required $(10,16)$. Indeed, vertical jump tests have been suggested as a measure of explosive strength; yet, the importance of RFD to vertical jump performance remains controversial (20). Previous researchers have reported trivial to very large correlations between RFD measured under isometric conditions and vertical jump height $(1,30)$. It is likely that the relationship will be influenced by the knee angle adopted during the isometric test 
and the type of vertical jump used. Here, Haff et al. (13) observed very large correlations between isometric RFD and jump height during a squat jump, but only trivial correlations between isometric RFD and jump height achieved during the CMJ. Similarly, on the same study, RFD recorded during dynamic strength exercises such as the mid-thigh pull also have shown to demonstrate only trivial-to-moderate correlations with jump height. Others have reported that RFD is not strongly related to the height achieved during a vertical jump, even when the measurement is recorded during the jump itself (16). Nevertheless, there is evidence that RFD is an important determinant of jumping performance, even when RFD is recorded during the propulsive phase of the jump (27). These findings could be because the subject's recruiter had no background with resistance training or experienced with explosive exercises (17). Given that the definition of explosive strength encompasses the ability to develop force rapidly, one would expect RFD to be strongly related to vertical jump performance if vertical jump is a valid measure of explosive strength. Presently, there is a scarcity of information regarding these relationships in a large group of trained athletes. Examination of these relationships could be of great importance for the optimal development of resistance training programs to improve vertical jumping performance in well-trained athletes. Furthermore, different measuring apparatuses have been used such as isoinertial devices (10), isometric devices (1), contact mats (28), linear encoders (20), and force platforms (10). Force platforms are the most common measuring device and are currently the gold standard for this type of assessment $(7,11,19,25)$. Data acquisition with this equipment, however, is time consuming, and complex calculation procedures make it inconvenient for use during training sessions. Linear transducers are frequently used alternatives as they are portable, affordable, easy to operate, and less time consuming than force platforms Hence, this equipment may be a good option for evaluating subjects for both training and research applications. Besides, linear transducer output seems to be highly associated with force when compared with those of a force platform (20). On this, linear transducers would enable investigators to determine the contribution of dynamic force and RFD and velocity to displacement during a vertical jump. Furthermore, the linear transducer has shown high validity and reliability in measurements of force when compared with a force platform. Therefore, the goal of this study was to determine the association between force-time measures and CMJ performance collected with a linear transducer. Based on what was explained earlier, it was hypothesized that there should exist an association between force-time parameters (force, dynamic RFD, velocity, and displacement and $\mathrm{CMJ}$ performance).

\section{MethodS}

\section{Experimental Approach to the Problem}

It was selected a cross-sectional experimental design to determine the hypothetical association between force-time measures and $\mathrm{CMJ}$ performance in a large group of trained male athletes. All testing was performed at the completion of the second period of in-season training in 1 session.

\section{Subjects}

A group of 35 male trained subjects (age range 21-27 years) volunteered to participate in the study (mean $\pm S D$ : age $24.3 \pm 1.5$ years, body mass $71.5 \pm 4.4 \mathrm{~kg}$, body height $1.76 \pm 0.02 \mathrm{~m})$. All participants were trained amateur athletes of different sports (e.g., soccer, indoor soccer, track \& field, and team handball). Each athlete competed at National and International level events during this period and had experience with resistance training. Apart from normal technical and tactical practice sessions (2-3 hours per day timed for 4:00 PM) and weekend competitions, all volunteers were involved in a 16 -week resistance training program. Two to 3 resistance training sessions were completed per week and included 2 maximal dynamic strength exercises (bench press and half squat) and 3 explosive exercises, such as cleans, snatch, and weighted CMJs. Consequently, all the athletes were highly trained and familiar with the testing exercise.

Before the data collection, subjects had a physical examination, and each was cleared of any medical disorders that might limit full participation in the investigation. The participants were informed of the purpose and method of the study to ensure that they understood completely, and each provided written informed consent to participate. The study was approved by the ethics committee of University of Beira Interior in Portugal and was conducted in accordance with the Declaration of Helsinki for Human Research.

\section{Procedures}

After a standard warm-up, participants performed 3 maximal $\mathrm{CMJ}$ trials in a Smith machine. The bar of this apparatus had a linear transducer attached (T-FORCE, Murcia, Spain). The rotary encoder of the linear transducer recorded the position and direction of the bar (weighted $17 \mathrm{~kg}$ ) to within an accuracy of $0.0002 \mathrm{~m}$. Only the concentric portion of the $\mathrm{CMJ}$ was taken to analysis. Concentric movement was defined from the moment after the end of the eccentric phase until maximal positive velocity was achieved. Vertical instantaneous velocity (v) was directly measured by the device and sampled at a frequency of $1,000 \mathrm{~Hz}$. The linear transducer was interfaced with a personal computer by means of a 14-bit resolution analog-to-digital data acquisition board, where a specialized software (T-FORCE Dynamic Measurement System) application automatically calculates the relevant kinematic and kinetic parameters of every jump, provides real time information on screen, and registers all data on disk for subsequent analysis. The derived mechanical variables were calculated by the software as follows: displacement was obtained by integration of $\mathrm{v}$ data with respect to time; instantaneous acceleration (a) was obtained from differentiation of $\mathrm{v}$ with respect to time; instantaneous force $(F)$ was calculated as $F=m(a+g)$, where $\mathrm{m}$ is the moving mass (in kilograms) that must be 
manually entered into the software for each set, and $g$ is the acceleration due to gravity $\left(9.81 \mathrm{~m} \cdot \mathrm{s}^{-2}\right)$. Because the effect of friction force was negligible in pilot testing, it was not taken into consideration in the calculations. Similarly excluded from consideration was the constant downward force exerted by the cable because it was minimal compared with the weight being lifted. The validity and reliability of this system have been previously established (20). The CV ranged from 3.6 to $17 \%$, with the values being greater for the RFD measures, while the ICC values ranged from 0.93 to 0.98 with the lowest value being recorded for RFD at peak force $(\mathrm{PF})$.

\section{Statistical Analyses}

Mean $( \pm S D)$ were calculated for each variable. The normality and homoscedasticity assumptions were checked respectively with the Shapiro-Wilk and the Levene Tests. The ICC was used to determine between-subject reliability of jumping tests. Within-subject variations for all tests were determined by calculating the $\mathrm{CV}$ as outlined by Hopkins (15). Pearson product-moment correlation coefficient was used to verify the association between variables. The level of significance was set at $p \leq 0.05$.

\section{Results}

Countermovement jump mechanical parameters are presented in Table 1. Pearson product-moment correlation coefficients between jump height and strength metrics of the CMJ are presented in Table 2. This study demonstrated high ICC values indicating that each variable has high capacity to differentiate among subjects. Concurrently, within-subject variation, as indicated by the coefficient of variation $\mathrm{CV}$, was somewhat high (Table 1 ).

TABLE 1. Intraclass correlation coefficient (ICC) and coefficients of variation (CV \%) for the CMJ as measured by the linear transducer and Optojump system simultaneously.

\begin{tabular}{lcc}
\hline & $\begin{array}{c}\text { ICC } \\
\text { (range) }\end{array}$ & $\begin{array}{c}\text { CV } \\
(\%)\end{array}$ \\
\hline $\begin{array}{l}\text { Linear transducer } \\
\text { parameters } \\
\text { Concentric peak } \\
\text { force (N) }\end{array}$ & $0.98(0.96-0.99)$ & 4.2 \\
$\begin{array}{l}\text { Time at peak force } \\
\text { (ms) }\end{array}$ & $0.92(0.87-0.96)$ & 18.1 \\
$\begin{array}{l}\text { Maximum rate of } \\
\text { force development } \\
\left.\text { (N.s }{ }^{-1}\right)\end{array}$ & $0.94(0.88-0.97)$ & 17.1 \\
$\begin{array}{l}\text { Optojump system } \\
\text { parameter } \\
\text { Jump height (m) }\end{array}$ & $0.97(0.92-0.98)$ & 4.4 \\
Flight time $(\mathrm{s})$ & $0.98(0.93-0.99)$ & 4.1 \\
\hline
\end{tabular}

TABLE 2. Linear relationships between CMJ height and distinct measures of strength.*

\begin{tabular}{|c|c|c|}
\hline & CMJ 1 & CMJ 2 \\
\hline Parameters & $r$ & $r$ \\
\hline $\operatorname{RFD}_{\max }\left(\mathrm{N} \cdot \mathrm{s}^{-1}\right)$ & $0.807 \dagger$ & $0.809 \dagger$ \\
\hline Time at RFD $\max (\mathrm{ms})$ & $-0.774 \dagger$ & $-0.791 \dagger$ \\
\hline Force at $\mathrm{RFD}_{\max }(\mathrm{N})$ & $-0.473 \ddagger$ & $-0.485 \S$ \\
\hline Velocity at $\operatorname{RFD}_{\max }\left(\mathrm{m} \cdot \mathrm{s}^{-1}\right)$ & NS & NS \\
\hline Displacement at RFD ${ }_{\max }(\mathrm{m})$ & NS & NS \\
\hline$\%$ of $P F$ at $\mathrm{RFD}_{\max }(\mathrm{N})$ & $-0.823 \dagger$ & $-0.809 \dagger$ \\
\hline $\mathrm{PF}(\mathrm{N})$ & $-0.629 \dagger$ & $-0.614 \dagger$ \\
\hline Time at $P F\left(m \cdot s^{-1}\right)$ & $-0.783 \dagger$ & $-0.849 \dagger$ \\
\hline $\mathrm{RFD}$ at $\mathrm{PF}\left(\mathrm{N} \cdot \mathrm{s}^{-1}\right)$ & $0.787 \dagger$ & $0.779 \dagger$ \\
\hline Velocity at PF $\left(\mathrm{m} \cdot \mathrm{s}^{-1}\right)$ & $0.491 \S$ & $0.414 \S$ \\
\hline
\end{tabular}

${ }^{*} \mathrm{CMJ}=$ countermovement jump; $\mathrm{RFD}_{\max }=$ maximal rate of force development; NS = nonsignificant; PF = peak force.

$\dagger$ Significance $p<0.001$

$¥$ Significance $p<0.01$.

§Significance $p \leq 0.05$
The current experiment found important relationships between CMJ displacement performed with a $17 \mathrm{~kg}$ external load and several parameters measured with the linear transducer (Table 2). This research showed significant correlation between $\mathrm{CMJ}$ displacement and the maximum dynamic RFD $(r=0.81, p<0.01)$ and between the CMJ performance and the percentage of the $\mathrm{PF}(r=-0.81$ and $-0.82, p<0.01$ for CMJ1 and CMJ2, respectively) at $\mathrm{RFD}_{\text {max }}$. The negative association suggests that as jump displacement improves, the percentage of the PF likewise tends to diminish. The same trend was verified for the association between the CMJ displacement and the inverted time to reach an $\mathrm{RFD}_{\max }$ as well as the displacement and the RFD produced at PF. Moreover, it was shown that CMJ displacement was related with both force attained in $\mathrm{RFD}_{\text {max }}$. Velocity and displacement at $\mathrm{RFD}_{\max }$ were not significantly associated with the weighted bar CMJ.

\section{Discussion}

The purpose of this study was to investigate the association between force-time parameters (force, dynamic RFD, velocity, and displacement) and CMJ performance in resistance trained subjects. The percentage of force at maximum RFD was the most important variable to better explain jump performance (i.e., movements involving high acceleration). To our best knowledge, this is one of the few studies that examined with so much depth the variables that can explain the CMJ performance in athletes as the one that present here. This study has had the worry to measure with instrumental severity and with highly reliability in the different variables. Moreover, the number of subjects has 
been considerable (15), and in trained sportsmen population, what permits us to have greater confidence in the results.

It has been reported that significant correlations were observed between the isometric RFD and vertical jump $(21,29)$, while others obtained antithetical readings (31). The findings of this study suggest that CMJ performance is significantly related to $\mathrm{RFD}_{\max }$ and other parameters related to RFD, such as the time to reach the displacement at $\mathrm{RFD}_{\text {max }}$ (from $r=-0.79$ to $-0.77, p<0.01$ ); and the RFD attained at PF $(r=0.78-0.79, p<0.01)$. The selection of different measuring devices (with different accuracy levels) and muscle tensions (i.e., isometric vs. dynamic) may explain the mixed findings $(1,18,19)$. Confounding factors such as body balance or familiarization with the protocol may have contributed to the mixed findings.

The PF measured with the linear transducer was also significantly associated with the CMJ performance $(r=0.614, p<0.001)$. González-Badillo and Marques (12) suggested that concentric $\mathrm{PF}$ was significantly related with CMJ performance $(r=0.70-0.82, p<0.001)$. However, Cordova and Armstrong (8) did not observe associations between the PF applied with a force platform and the single leg jump performance. Although they obtained a high ICC $(\mathrm{ICC}=0.94)$, the $\mathrm{CV}$ was very high, which one might consider as representative of a lower or poor reliability (23). Additionally, Cordova and Armstrong (8) selected different protocols to measure jump performance (e.g., they only measured the jump with the right leg). For the present investigation, PF data were quite reliable (ICC: 0.99 and CV: $3.6 \%)$.

More interesting was the association between the percentage of force at $\mathrm{RFD}_{\max }$ and CMJ performance $(r=-0.823 /-0.09, p<0.001)$. To the authors' best knowledge, no previous study has selected and reported this parameter. This relationship could be simultaneously explained by 2 main factors. First, subjects tend to jump higher when they produce more force during the concentric phase (12). Furthermore, this study demonstrated that the greater the jump displacement, the lower will be the time to achieve the $\mathrm{RFD}_{\text {max }}$ (from $r=-0.77$ to $-0.79, p<0.001$ ). Thus, the CMJ performance tends to be higher when the $\mathrm{RFD}_{\text {max }}$ is produced sooner and the PF produces higher values (i.e., the $\mathrm{PF}$ attained at $\mathrm{RFD}_{\max }$ tends to be lower). In others words, if the percentage of the peak of force applied at the moment of attaining the $\mathrm{RFD}_{\max }$ are reduced, the height of the jump likewise will be higher. Here, it is important that the force produced in the first milliseconds of the concentric jump phase must be maintained at high values of correlation with $\mathrm{CMJ}$ displacement carried out by the subjects (12). In addition, these authors also verified that concentric $\mathrm{PF}$ was significantly related with $\mathrm{CMJ}$ performance for each trial (from $r=0.70-0.82, p<0.001$ ). As reported elsewhere (32), the start gradient is the RFDs' initial $50 \%$ of time required to reach $\mathrm{PF}$, while the second $50 \%$ is the acceleration gradient; so, this finding might be related to such events.

Neither velocity nor displacement at $\mathrm{RFD}_{\max }$ during the concentric phase of the jump presented significant associations with the CMJ. This lack variability of is probably a "statistical" artefact because both velocity and displacement attained at the $\mathrm{RFD}_{\text {max }}$ are very small and very similar in all subjects. This lack of reliability reduces the possibility of a high correlation between them. What differentiate subjects are the force produced and the time taken in reaching it not the displacement or the velocity by which the $\mathrm{RFD}_{\max }$ is reached. To our knowledge, no previous study has analyzed these 2 variables as possible predictors of vertical jump displacement. However, time taken to reach the $\mathrm{RFD}_{\max }$ showed a strong correlation $(r=-0.791$ and -0.774 , $p<0.001)$ with CMJ performance. This finding highlights how the RFD is important to those sports in which reaching high acceleration over very short periods of time is necessary. In theory, the changes in CMJ performances and the changes in the time spent to produce force should present an important negative association, as demonstrated in this study. However, if we consider the time taken to reach PF, the correlation was also negative and significant $(r=-0.78$ / $-0.84, p<0.001)$ as well as the RFD at PF $(r=-0.79 /$ $-0.78, p<0.001)$.

In several explosive sports events, athletes must improve jump performance (i.e., stretch-shortening cycle) to excel. Jump performance tests can be selected to assess one's adaptations to a given training regime, including fitness- or performance-oriented subjects $(5,28)$. It can also be used for talent identification, development, and follow-up of young athletes (14). One of the findings of this research was that dynamic RFD is one of the most important variables to explain jump performance. This has obvious application to sports dealing with force production that takes between 100 and $300 \mathrm{~ms}$ as the ones involving skills such as sprinting, throwing, and jumping. Therefore, $\mathrm{S} \& \mathrm{C}$ coaches should design training sessions accordingly.

Based on the current results, it is possible that the peak bar velocity is an important factor to considerate to develop the short-sprint performance in trained athletes. Thus, it is suggested that sprinting time performance would benefit from training regimens aimed to improve these performance qualities. Furthermore, the great majority of research uses acyclic vertical type movements (e.g., squat, vertical jumps) to predict an activity that is cyclic and horizontal in nature. Further research may benefit from investigating movements that require greater horizontal force production.

\section{Practical Applications}

Improvement in short distance sprint ability is a major training goal for many sports, and countermovement jumping is a well-rounded training exercises to build-up this fitness component. In sports such as basketball, soccer, or 
team handball, players must improve sprinting performance over very short distances.

The maximal RFD was strongly related to $\mathrm{CMJ}$ displacement and also to the percentage of $\mathrm{PF}$ at $\mathrm{RFD}_{\text {max }}$. The percentage of $\mathrm{PF}$ applied at $\mathrm{RFD}_{\max }$ and $\mathrm{RFD}_{\max }$ were the best predictive variables for $\mathrm{CMJ}$ performance. Therefore, $\mathrm{S} \& \mathrm{C}$ practitioners should consider monitoring such parameters on a regular basis. An affordable and straightforward method is to use a linear encoder. Indeed, this piece of equipment can also be used as a biofeedback system because the athlete himself and the coach might be watching on the screen the force-time curve being displayed. This will provide (a) an evidence-based practice, (b) a deeper insight about the acute response to the training routine, (c) one might say that also the biofeedback system is playing as an external motivation, and (d) to be an effective way to build-up explosiveness and enhance the CMJ performance or any other motor skill that involves explosive strength.

Having said that these findings should be interpreted with caution because correlations do not signify causation, additional research is required to clarify whether improvements in upper body strength or velocity as a result of resistance and/or plyometric training will indeed improve jumping ability in trained track and field athletes.

\section{ACKNowledgments}

The authors thank the dedicated group subjects who participated in this study.

\section{REFERENCES}

1. Abernethy, P, Wilson, G, and Logan, P. Strength and power assessment: Issues, controversies and challenges. Sports Med 19: 401-417, 1995.

2. Aragon-Vargas, LF and Gross, M. Kinesiological factors in vertical jump performance: Differences among individuals. J Appl Biomech 13: 24-44, 1997.

3. Aragon-Vargas, LF and Gross, M. Kinesiological factors in vertical jump performance: Differences within individuals. J Appl Biomech 13: 45-65, 1997.

4. Ashley, CD and Weiss, LW. Vertical jump performance and selected physiological characteristics of women. J Strength Cond Res 8: 5-11, 1994.

5. Baker, D. Improving vertical jump performance through general, special, and specific strength training: A brief review. J Strength Cond Res 10: 131-136, 1996.

6. Bobbert, MF and Van Ingen Schenau, GJ. Coordination in vertical jumping. J Biomech 21: 249-262, 1988.

7. Carlock, JM, Smith, SL, Hartman, MJ, Morris, RT, Ciroslan, DA, Pierce, KC, Newton, RU, Harman, EA, Sands, WA, and Stone, MH. The relationship between vertical jump power estimates and weightlifting ability: A field-test approach. $J$ Strength Cond Res 18: 534-539, 2004.

8. Cordova, ML and Armstrong, CW. Reliability of ground reaction forces during a vertical jump: Implications for functional strength assessment. J Athl Train 31: 342-345, 1996.

9. Cronin, JB and Hansen, KT. Strength and power predictors of sports speed. J Strength Cond Res 19: 349-357, 2005.

10. Cronin, JB, Hing, RD, and McNair, PJ. Reliability and validity of a linear position transducer for measuring jump performance. J Strength Cond Res 18: 590-593, 2004.
11. Dowling, JJ and Vamos, L. Identification of kinetic and temporal factors related to vertical jump performance. J Appl Biomech 9: 95-110, 1993.

12. González-Badillo, JJ and Marques, MC. Relationship between kinematic factors and countermovement jump height in trained track and field athletes. J Strength Cond Res 24: 3443-3447, 2010.

13. Haff, GG, Stone, M, O’Bryant, HS, Harman, E, Dian, C, Johnson, R, and Han, KH. Force-time dependent characteristics of dynamic and isometric muscle actions. J Strength Cond Res 11: 269-272, 1997.

14. Hoare, DG and Warr, CR. Talent identification and women's soccer: An Australian experience. J Sports Sci 18: 751-758, 2000.

15. Hopkins, WG. Measures of reliability in sports medicine and science. Sports Med 30: 1-15, 2000.

16. Kawamori, N and Haff, GG. The optimal training load for the development of muscular power. J Strength Cond Res 18: 675-684, 2004.

17. Kawamori, N, Rossi, SJ, Justice, BD, Haff, EE, Pistilli, E, O'bryant, HS, Stone, MH, and Haff, GG. Peak force and rate of force development during isometric and dynamic mid-thigh clean pulls performed at various intensities. J Strength Cond Res 20: 483-491, 2006.

18. Markovic, G, Dizdar, D, Jukic, I, and Cardinale, M. Reliability and factorial validity of squat and countermovement jump tests. J Strength Cond Res 18: 551-555, 2004.

19. Marques, MC, Gil, H, Ramos, RJ, Costa, A, and Marinho, D. Relationships between vertical jump strength metrics and 5 meters sprint time. J Hum Kinet 29: 115-122, 2011.

20. Marques, MC and González-Badillo, JJ. Relationship between strength parameters and squat jump performance in trained athletes. Motricidade 7: 43-48, 2011.

21. Matavulj, DL, Kukolj, M, Ugarkovic, D, Tihanyi, J, and Jaric, S. Effects of plyometric training on jumping performance in junior basketball players. J Sports Med Phys Fitness 41: 159-164, 2001.

22. Moir, G, Button, C, Glaister, M, and Stone, MH. Influence of familiarization on the reliability of vertical jump and acceleration sprinting performance in physically active men. $J$ Strength Cond Res 18: 276-280, 2004.

23. Moir, G, Shastri, P, and Connaboy, C. Intersession reliability of vertical jump height in women and men. J Strength Cond Res 22: 1779-1784, 2008.

24. Murphy, AJ and Wilson, GJ. Poor correlations between isometric tests and dynamic performance: Relationship to muscle activation. Eur J Appl Physiol 73: 353-357, 1996.

25. Nigg, BM and Herzog, W. Biomechanics of the Muscle-Skeletal System. Chichester, United Kingdom: John Wiley \& Sons, 1994.

26. Rehn, B, Lidstrom, J, Skoglund, J, and Lindstrom, B. Effects on leg muscular performance from whole-body vibration exercise: A systematic review. Scand J Med Sci Sports 17: 2-11, 2007.

27. Sleivert, $\mathrm{G}$ and Taingahue, $\mathrm{M}$. The relationship between maximal jump-squat power and sprint acceleration in athletes. Eur J Appl Physiol 91: 46-52, 2004.

28. Vescovi, JD, Rupf, R, Brown, TD, and Marques, MC. Physical performance characteristics of high-level female soccer players 12-21 years of age. Scand J Med Sci Sports 21: 670-678, 2011.

29. Vitasalo, JT and Aura, O. Seasonal fluctuation of force production in high jumpers. Can J Appl Sport Sci 9: 209-213, 1984.

30. Wilson, GJ, Lyttle, AD, Ostrowski, KJ, and Murphy, AJ. Assessing dynamic performance: A comparison of rate of force development tests. J Strength Cond Res 9: 176-181, 1995.

31. Young, WB and Bilby, GE. The effect of voluntary effort to influence speed of contraction on strength, muscular power, and hypertrophy development. J Strength Cond Res 7: 172-178, 1993.

32. Zatsiorsky, VM. Science and Practice of Strength Training. Champaign, IL: Human Kinetics Books, 1995. 